\title{
Multiple Metastasis-Like Bone Lesions in Scintigraphic Imaging
}

\author{
Ying Zhang, 1, 2,3,4 Chunlei Zhao, 1, 2,3, 4, 5 Hongbiao Liu, 1, 2, 3, 4 \\ Haifeng Hou, 1, 2, 3,4 and Hong Zhang 1, 2, 3,4 \\ ${ }^{1}$ Department of Nuclear Medicine, Second Affiliated Hospital of Zhejiang University School of Medicine, Hangzhou, \\ Zhejiang 310009, China \\ ${ }^{2}$ Zhejiang University Medical PET Center, Hangzhou 310009, China \\ ${ }^{3}$ Institute of Nuclear Medicine and Molecular Imaging, Zhejiang University, Hangzhou 310009, China \\ ${ }^{4}$ Key Laboratory of Medical Molecular Imaging of Zhejiang Province, Hangzhou 310009, China \\ ${ }^{5}$ Department of Nuclear Medicine, Hangzhou First People's Hospital, Hangzhou Cancer Hospital, Hangzhou 310009, China
}

Correspondence should be addressed to Hong Zhang, hzhang21@gmail.com

Received 1 January 2012; Accepted 20 January 2012

Academic Editor: Mei Tian

Copyright ( $) 2012$ Ying Zhang et al. This is an open access article distributed under the Creative Commons Attribution License, which permits unrestricted use, distribution, and reproduction in any medium, provided the original work is properly cited.

\begin{abstract}
Multiple benign osteolytic lesions are very hard to differentiate from disseminated bone metastasis. Whole-body bone scintigraphy (WBBS) with technetium-99m methylene diphosphonate (Tc-99m MDP) demonstrates multiple lesions with increased uptake in any bone involved. Even combined with medical history and multiple imaging results, such as MRI and CT, the clinical diagnosis of metastasis lesion remains as a challenge. These clinical characteristics are similar to multiple malignant bone metastases and therefore affect the following treatment procedures. In this paper, we analyzed multiple benign osteolytic lesions, like eosinophilic granuloma (EG), multiple myeloma (MM), disseminated tuberculosis, fibrous dysplasia, or enchondroma, occurring in our daily clinical work and concluded that additional attention should be paid before giving the diagnosis of multiple bone metastases.
\end{abstract}

\section{Introduction}

Radioisotopes play an important role in the diagnosis of benign and malignant bone lesions. Whole-body bone scintigraphy (WBBS) with technetium-99m methylene diphosphonate (Tc-99m MDP) is widely available and commonly used to detect metastases from malignant tumors because it is more sensitive than X-rays and allows the whole body to be surveyed. However, multiple benign osteolytic lesions are very similar with bone metastasis in findings of bone scintigraphy. Before sending out the report of multiple metastases, benign osteolytic lesions should be considered according to other imaging findings, detailed medical history, or even the preliminary pathology result.

In this pictorial review, we retrospectively studied the WBBS with Tc-99m MDP findings in 5 patients of selected multiple benign osteolytic lesions. For adult patients, $15 \mathrm{mCi}$ Tc-99m MDP was administered intravenously. For pediatric patients, a smaller dose was used dependent on patient body weight: adult dose/70 $*$ patient weight $(\mathrm{kg})$.

\section{Eosinophilic Granuloma}

A 41-year-old man with left upper arm pain for 3 months was admitted for left humerus fracture after a tumble. All of images of Tc-99m MDP WBBS (Figure 1), left shoulder joint MRI (Figure 2), and thoracic CT (Figure 3) indicated bone metastases. Left humerus resection and internal fixation were performed thereafter. EG was diagnosed definitely by histopathological examination (positive: CD1a, S-100, LCA, Ki67 (6\%) and negative: CD68, CD79a, CD138, and P53). The patient received 4 courses of chemotherapy (steroids, vinca alkaloids, and antimetabolites) before discharge. Two years later, the patient was admitted again in our hospital for right femoral fracture caused by an accidental fall. Another Tc-99m MDP WBBS (Figure 4) was performed, and similar 


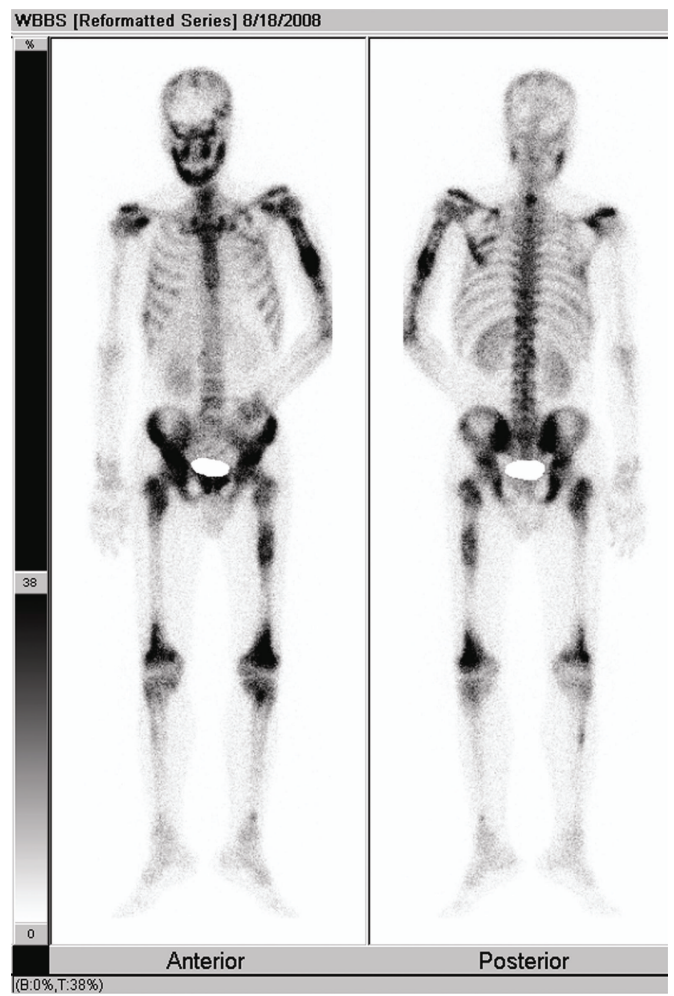

FIGURE 1: Tc-99m MDP WBBS of the first admission found multiple lesions with intensive radioactive accumulation in skull, mandible, left humerus, scapulas, ribs, vertebras, pelvis, femurs, and tibias.

findings (except for deterioration of right femur site) were observed as shown in the previous one (Figure 1).

\section{Multiple Myeloma}

A 45-year-old female patient suffered chest pain for 2 months that deteriorated recently. Blood routine examination found WBS $4.0 * 10^{9} / \mathrm{L}, \mathrm{Hb} 43 \mathrm{~g} / \mathrm{L}$ and Plt $243 * 10^{9} / \mathrm{L}$. Bone marrow biopsy demonstrated plasma cell myeloma. Serum immunoglobulin (IgG) and $\beta 2$-microglobulin (MG) were $43.59 \mathrm{~g} / \mathrm{L}$ and $18.58 \mathrm{mg} / \mathrm{L}$, respectively, and both were above the normal level. Tc-99m MDP WBBS (Figure 5) was performed before chemotherapy and found multiple bones accumulated the agent abnormally. Clinical diagnosis was multiple myeloma IgG stage III; then, chemotherapy of VAD regimen was given to this patient.

\section{Disseminated Tuberculosis}

A 25-year-old male patient suffered cough and chest pain for 1 month, and the pain extended to back and waist recently. Diffused military nodules with small cavities in both lungs, and multiple bone destructions in thoracic and lumbar vertebra were found on chest CT suggesting of disseminated tuberculosis. Tc-99m MDP WBBS (Figure 6) demonstrated high uptake in multiple bones. The patient felt remission after the treatment of antituberculosis drugs.

\section{Fibrous Dysplasia}

An 18-year-old female patient suffered right hip pain after long walking for this year. She received sectional resection of right femur and allograft for the reason of fibrous dysplasia when she was only 7 years old. X-ray showed benign tumor changes in upper section of right femur, considering fibrous dysplasia. WBBS (Figure 7) before operation found abnormal accumulation in right femur, tibia, and cotyle. Then operation of right femur tumor resection and internal fixation was performed.

\section{Enchondroma}

A 14-year-old male patient found swelling of interphalangeal joint in left middle finger 11 years ago. Then the swelling extended to left ring and little fingers. CR and MRI illustrated multiple enchondroma in left hand. Before the operation, Tc-99m MDP WBBS (Figure 8) found additional bones involvement. This patient received sectional resection of multiple lesions in left hand and allograft the next day. Histopathological examination after operation was cracked cartilage tissue which is in accordance with multiple enchondroma.

\section{Discussion}

EG, formerly termed histiocytosis $\mathrm{X}$, is the most common and benign form of the Langerhans cell histiocytosis which 

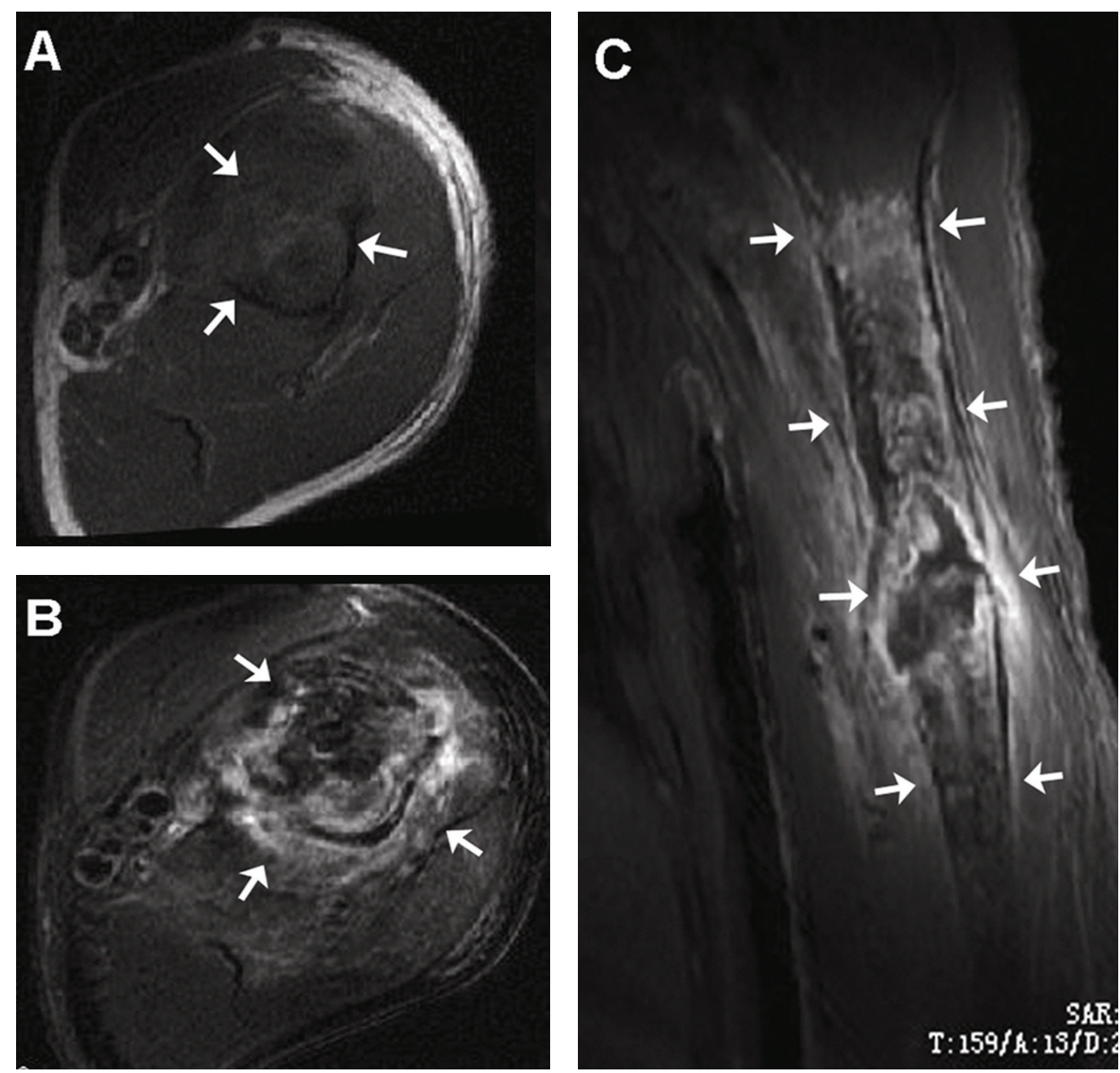

FIGURE 2: MRI of left shoulder demonstrated isointensity and mild-hypo-intensity signal on T1-weighted images (A), hyperintensity signal on T2-weighted images (B), and inhomogenily enhanced after Gd-DTPA administration (C) in humeral midshaft and surrounding soft tissues. These abnormal signals prompted bone malignancy and pathologic fracture.

includes another two forms: the Hand-Schüller-Christian disease (the triad of diabetes insipidus, exophthalmos, and lytic bone lesions) and the Letterer-Siwe syndrome (a rapidly-progressing multisystem disease with poor prognosis). EG predominantly affects children and adolescents although it can be found in any age. Solitary lesion is more common than multiple lesions. In our cases, a 41-year-old man with EG admitted for pathologic fracture demonstrated multiple malignant metastases like bone involvement on both scintigraphic and radiographic imaging. Although multiple bones were involved in this case with EG, we can easily differentiate it from the Hand-Schüller-Christian disease for the absence of main characteristics of diabetes insipidus and exophthalmos. The etiology of EG is still unknown. The skeletal involvement is frequent and generally demonstrates as destroyed solitary or multiple bones in any age [1-4], while the visceral involvement is rare and often occurs at age below 2 years old [5]. Localized pain is always the first symptom of bone involvement, but it can also be presented as pathologic fractures after injuries. The most commonly involved bones are skull, mandible, pelvis, spine, ribs, and long bones. Radiographic imaging is the main modality for the diagnosis of EG, although the radiographic characteristics of EG are considerably different according to the site and the activity of the lesion [6]. Bone destruction in granulation tissue forming areas of osteolysis is the main pathological characteristic of EG and may involve any portion of any bone [7].

Typical finding on Tc-99m MDP WBBS in the patients with EG is the lesion with intensive radioactive accumulation with a photopenic center [1-3], because the bone involvement in EG always begins from osteolysis that arise in the bone marrow cavity and following periosteal reaction. However, all the manifestations of the lesions with increased, decreased, or normal accumulation on Tc-99m MDP WBBS could be observed according to the amount of viable residual or reactive bone tissue. Those can significantly accumulate the radioactive tracer $[6,8]$. In EG, Tc-99m MDP WBBS is generally less sensitive than radiography in the detection of osseous lesions [6]. The findings in this patient confirmed this opinion. However, Tc-99m MDP WBBS may play roles in searching additional bone lesions and monitoring therapeutic response and is helpful when the radiographs are normal or equivocal [6]. In addition, Tc-99m 

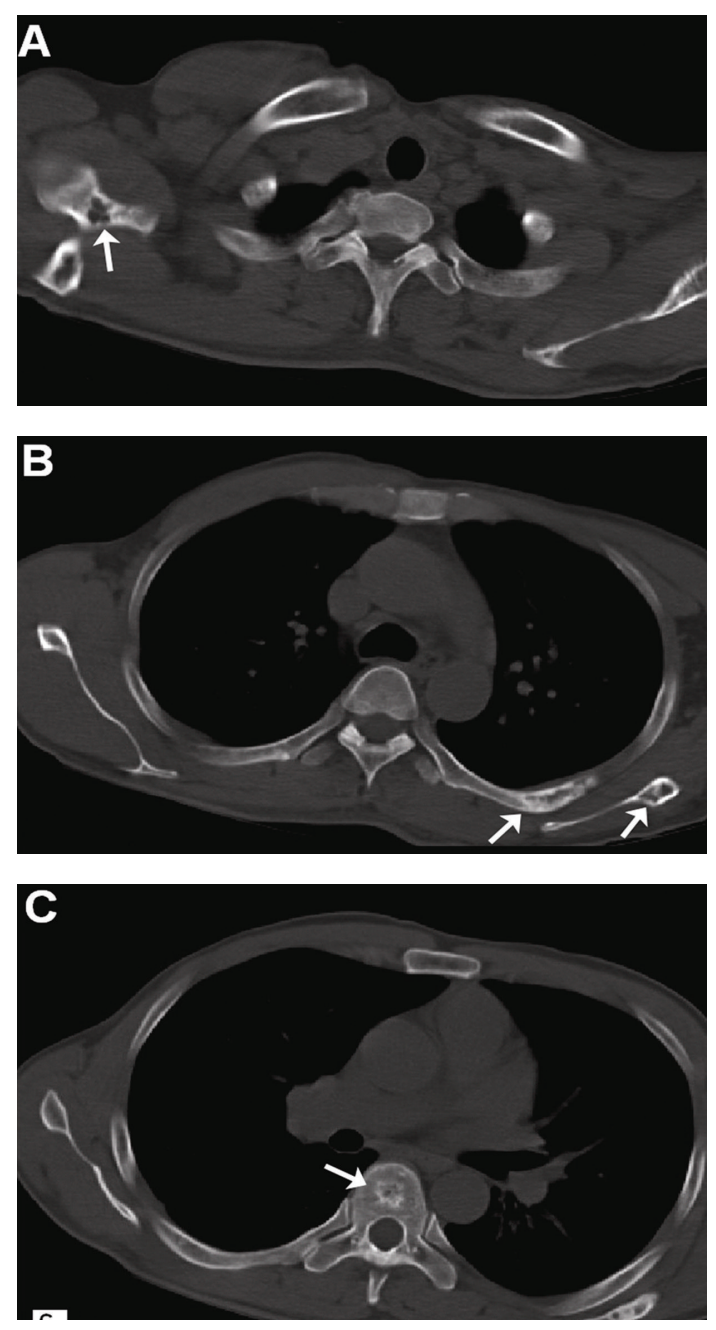

Figure 3: Chest CT of bone window showed eroded bone destruction with sclerotic edge in scapulas and ribs ((A): right scapula; (B): left scapula and the left 5 th posterior rib) and patchy high density in thoracic vertebrae (C) However, the amount of the lesions was greater than that in Tc-99m MDP WBBS within the corresponding thoracic area. The bone lesions on CT with intensive Tc-99m MDP accumulation prompted multiple bone metastases.

MDP WBBS has the advantage of detecting the asymptomatic bone abnormalities possibly missed by other imaging modalities. It is suggested that combination of various imaging modalities might be helpful to make a definite diagnosis when bone biopsy or operation is not available [9]. In this patient, all images of Tc-99m MDP WBBS, MRI, and CT demonstrated the characteristics of disseminated bone metastases although EG was finally diagnosed by postoperative pathological examination. Multiple bone lesions in EG are relatively rare because solitary lesion predominates over multiple lesions, and only around $10 \%$ of solitary lesion may eventually develop into multiple lesions [2]. EG is a benign inflammatory disease, and the activity of this disease may remain stable during follow-up [1]. In this case, the Tc-99m MDP WBBS performed 2 year later revealed only mild alternations in a wide range of bone involvement as shown in the previous one except the increased radioactive accumulation in fractured right femur, confirming the benignity of EG.

Multiple myeloma is a plasma cell dyscrasia of the bone marrow. It can be mistaken radiographically for metastatic bone disease because it presents with multiple osteolytic lesions or diffuse osteopenia. WBBS often fails to demonstrate a hyperconcentration at sites of skeletal involvement by myeloma despite extensive disease by radiography. This occurs because reactive bone formation is not a feature of the disease; this might be due to release of an inactivation factor by the tumor. Multiple myeloma is a common tumor of elderly patients. It is usually confirmed by serology or bone marrow examination. Radiographs typically show either a solitary or multifocal osteolytic pattern. However, skeletal survey is not always a useful screening method, so other imaging modalities such as CT and WBBS are often used. WBBS is sensitive in $75 \%$ of patients with myeloma, but only about $10 \%$ of lesions are shown [10]. Therefore, WBBS may be helpful when there is focal pain and a negative radiograph.

Skeletal TB occurs in only very small part of patients with $\mathrm{TB}$, and therefore the diagnosis is often difficult due to the nonspecific nature of the clinical presentation [11]. The spine, particularly the thoracolumbar spine, is the most common site of skeletal involvement of TB. Back pain can affect anyone and in general can originate from the bone (vertebrae), muscles, ligaments, tendons, or nerves. Tuberculosis of the spine is one of the causes for back pain, and early diagnosis and management can prevent complications. Imaging plays an important role in the diagnosis and management of spinal infections. Infection and inflammation of the spine are complex. The intense vertebra uptake on WBBS is nonspecific, so the differential diagnosis would include bone metastases from lung cancer [12]. It is important to obtain material for culture and sensitivity in the absence of confirmed pulmonary TB for a definitive diagnosis [13].

Fibrous dysplasia is a benign tumor in which masses of fibroblasts and islands of cartilage replace bone marrow in one or more locations. It is commonly found in young patients with mild bone pain or as an incidental finding, although the lesions may persist into late adulthood. The main value of WBBS in fibrous dysplasia is to show polyostotic involvement, but sometimes these lesions may show only minimal uptake or even no uptake [14]. From our case of multiple fibrous dysplasia, the differentiation from bone metastasis can be made by combining with their past medical history.

Enchondroma is a common benign lesion that appears in the medullary portion of bone and is composed chiefly of mature hyaline cartilage. The solitary enchondroma has a predilection for the phalanges and metacarpals of the hand. Multiple enchondromas, or enchondromatosis, is an anomaly usually disclosed in infancy. When one side of the body exhibits greater involvement than the other, the condition is called Ollier's disease. The combination of enchondromatosis and soft tissue hemangiomata is referred to as Maffucci's syndrome. Malignant transformation into chondrosarcoma can occur, especially in multiple enchondromatosis. Chondrosarcoma is exceedingly rare in the phalanges. 


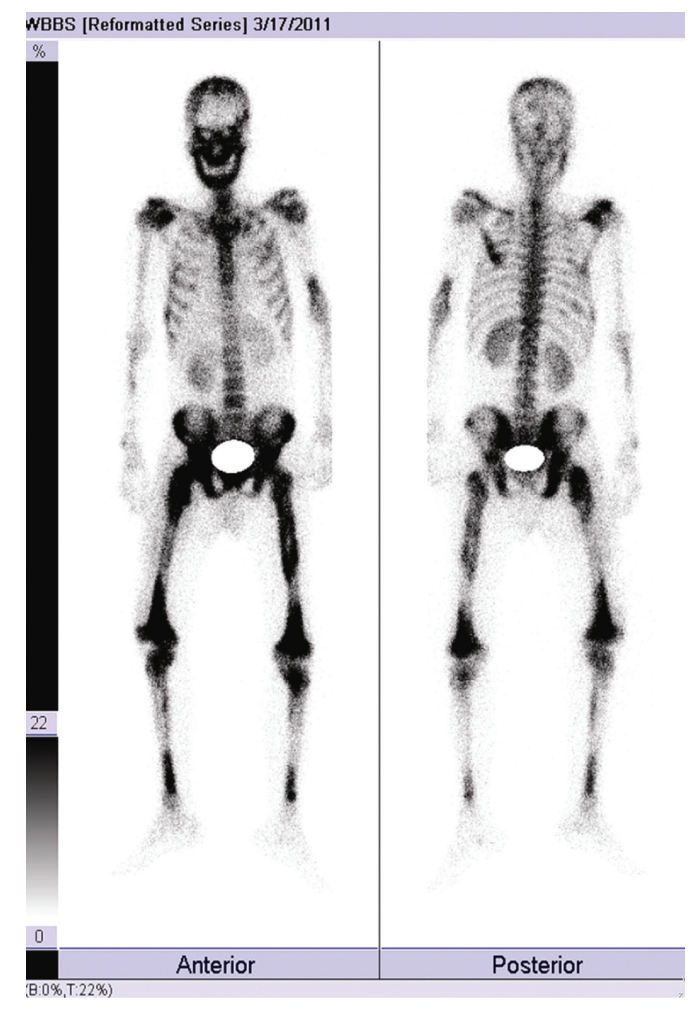

Figure 4: Tc-99m MDP WBBS of the second admission 2 years later showed multiple bone involvement observed as similar to those found in the previous Tc-99m MDP WBBS except the increased radioactive accumulation in fractured right femur.

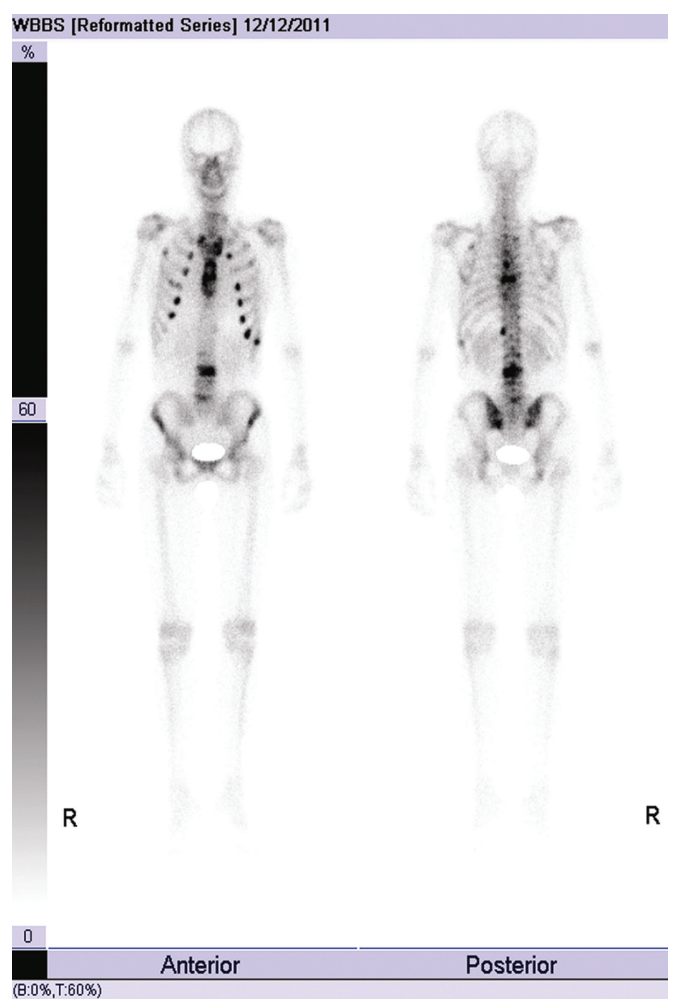

FIGURE 5: Tc-99m MDP WBBS before chemotherapy found a wide range of abnormal radioactive accumulation in ribs, thoracic and lumbar vertebra, sacrum, and pelvis. 


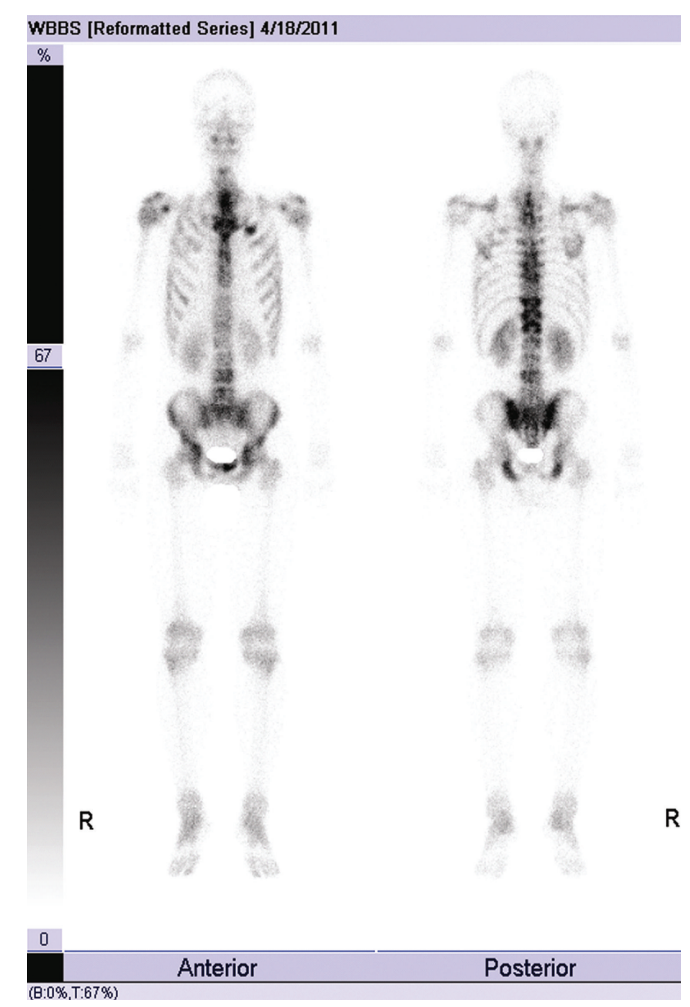

FIgURE 6: Tc-99m MDP WBBS showed ribs, spine, and pelvis intensively accumulated the agent and was very similar to the changes of multiple bone metastasis.

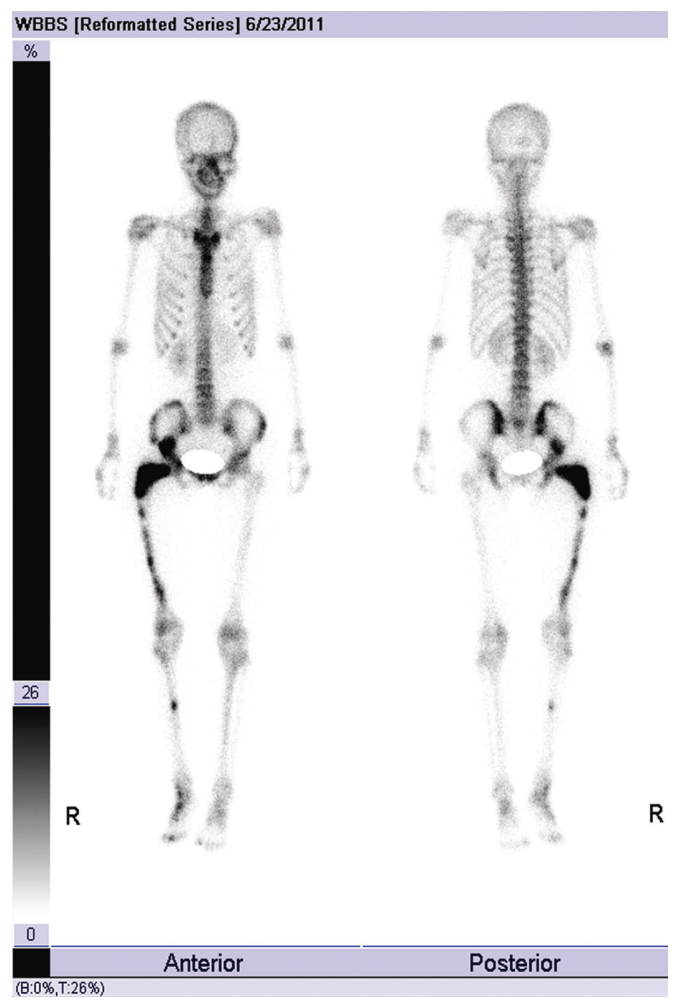

Figure 7: Tc-99m MDP WBBS found abnormal intensive accumulation in right femur, tibia, and cotyle suggesting tumor, and decreased accumulation in upper right femur due to the previous operation. 


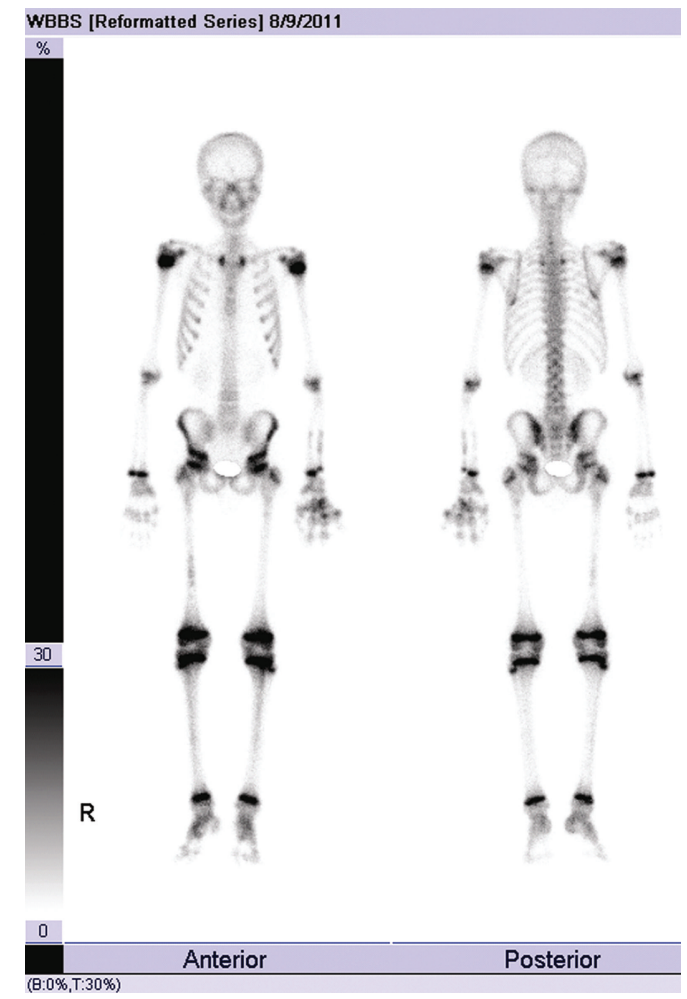

FIGURE 8: Tc-99m MDP WBBS before operation demonstrated intensive radioactive accumulation in multiple metacarpophalangeal and interphalangeal joints of left hand, left ulna, left radius, and right femur.

Enchondroma can have high FDG and MDP uptake on scintigraphic imaging and occasionally may mimic metastatic lesions in patients being screened for metastatic disease [15]. Taking one with another, WBBS is an effective and economic method in detecting multiple enchondromatosis.

\section{Conclusion}

WBBS is that of intense radioactive imaging agent uptake in the osteolytic lesions. Given the low specificity of Tc99m MDP WBBS and its sensitivity to increased bone remodeling from any cause, whether benign or malignant, the presence of multiple benign osteolytic lesions renders the interpretation indistinguishable from malignant metastasis. Therefore, we should be watchful of giving the diagnosis of disseminated bone metastasis during our daily work; furthermore, detailed past medical history and various laboratory results might be helpful for accurate diagnosis.

\section{Acknowledgments}

This work is partly sponsored by Grants from the Zhejiang Provincial Natural Science Foundation of China (Z2110230), Health Bureau of Zhejiang Province (2010ZA075, 2011ZDA013), National Science Foundation of China (NSFC) (no. 81101023, 81170306, 81173468), and Ministry of Science and Technology of China (2011CB504400, 2012BAI13B06).

\section{References}

[1] L. G. Flores, H. Hoshi, S. Nagamachi et al., "Thallium-201 uptake in eosinophilic granuloma of the frontal bone: comparison with technetium-99 m-MDP imaging," Journal of $\mathrm{Nu}$ clear Medicine, vol. 36, no. 1, pp. 107-110, 1995.

[2] M. Yoshikawa, Y. Sugawara, T. Kikuchi et al., "Two cases of pediatric bone disease (Eosinophilic Granuloma and Brodie's abscess) showing similar scintigraphic and radiographic findings," Clinical Nuclear Medicine, vol. 25, no. 12, pp. 986-990, 2000.

[3] S. Solav, "Bone Scintiscanning in Osteolytic Lesions," Clinical Nuclear Medicine, vol. 29, no. 1, pp. 12-20, 2004.

[4] B. D. Nguyen, M. C. Roarke, and S. F. Chivers, "Multifocal Langerhans cell histiocytosis with infiltrative pelvic lesions: PET/CT imaging," Clinical Nuclear Medicine, vol. 35, no. 10, pp. 824-826, 2010.

[5] J. Donadieu, "A multicentre retrospective survey of Langerhans' cell histiocytosis: 348 cases observed between 1983 and 1993," Archives of Disease in Childhood, vol. 75, no. 1, pp. 1724, 1996.

[6] K. Wang, L. Allen, E. Fung, C. C. Chan, J. C. S. Chan, and J. F. Griffith, "Bone scintigraphy in common tumors with osteolytic components," Clinical Nuclear Medicine, vol. 30, no. 10, pp. 655-671, 2005. 
[7] G. B. Greenfield, Radiology of Bone Disease, Lippincott Company, Philadelphia, Pa, USA, 3rd edition, 1980.

[8] S. Antonmattei, M. R. Tetalman, and T. V. Lloyd, "The multiscan appearance of eosinophilic granuloma," Clinical Nuclear Medicine, vol. 4, no. 2, pp. 53-55, 1979.

[9] R. Naumann, B. Beuthien-Baumann, R. Fischer et al., "Simultaneous occurrence of Hodgkin's lymphoma and eosinophilic granuloma: a potential pitfall in positron emission tomography imaging," Clinical Lymphoma, vol. 3, no. 2, pp. 121-124, 2002.

[10] W. Dahnert, "Bone and soft-tissue disorders," in Radiology Review Manual, W. Dahnert, Ed., vol. 122, Lippincott Company, Philadelphia, Pa, USA, 5th edition, 2002.

[11] L. Cormican, R. Hammal, J. Messenger, and H. J. Milburn, "Current difficulties in the diagnosis and management of spinal tuberculosis," Postgraduate Medical Journal, vol. 82, no. 963, pp. 46-51, 2006.

[12] N. Khattry, S. Thulkar, A. Das, S. A. Khan, and S. Bakhshi, "Spinal tuberculosis mimicking malignancy: atypical imaging features," Indian Journal of Pediatrics, vol. 74, no. 3, pp. 297298, 2007.

[13] A. I. de Backer, K. J. Mortelé, I. J. Vanschoubroeck et al., "Tuberculosis of the spine: CT and MR imaging features," Journal Belge de Radiologie, vol. 88, no. 2, pp. 92-97, 2005.

[14] J. Han, J. S. Ryu, M. J. Shin, G. H. Kang, and H. K. Lee, "Fibrous dysplasia with barely increased uptake on bone scan: a case report," Clinical Nuclear Medicine, vol. 25, no. 10, pp. 785-788, 2000.

[15] N. Dobert, C. Menzel, R. Ludwig et al., "Enchondroma: a benign osseous lesion with high F-18 FDG uptake," Clinical Nuclear Medicine, vol. 27, no. 10, pp. 695-697, 2002. 


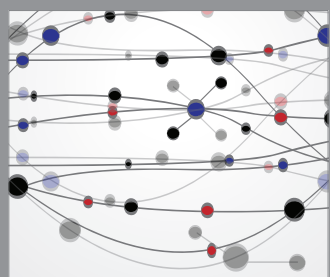

The Scientific World Journal
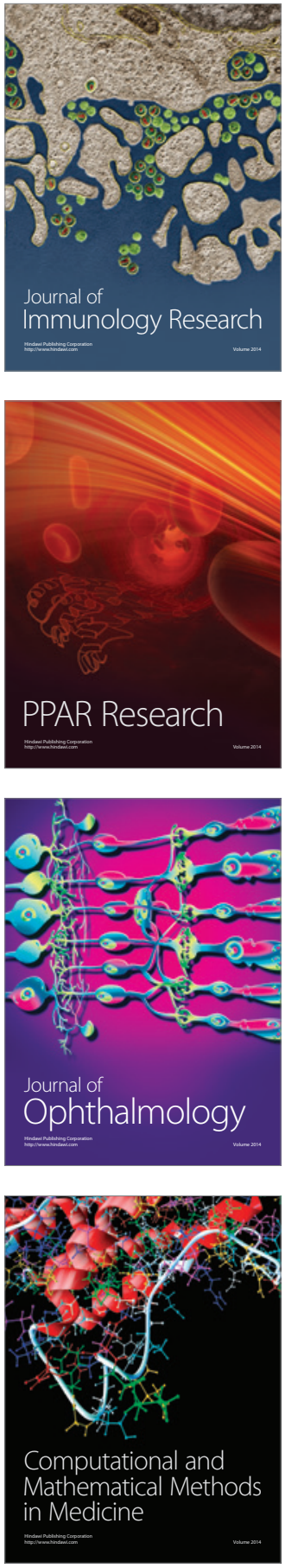

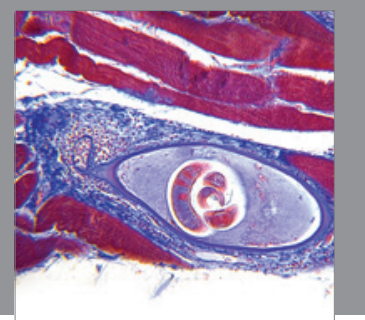

Gastroenterology

Research and Practice
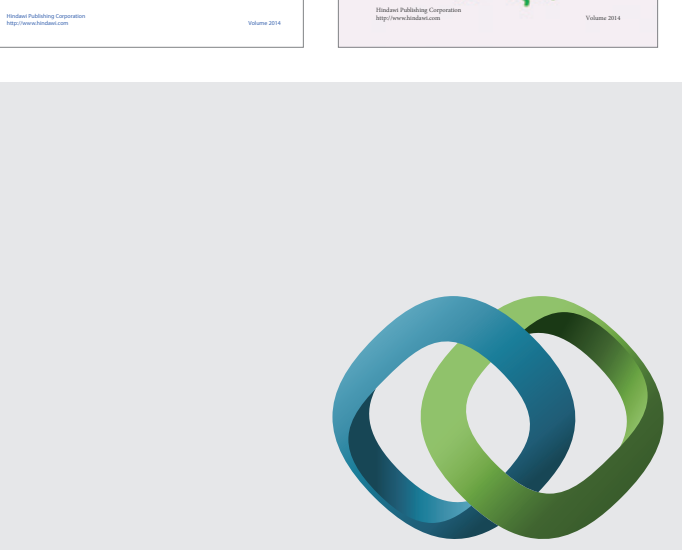

\section{Hindawi}

Submit your manuscripts at

http://www.hindawi.com
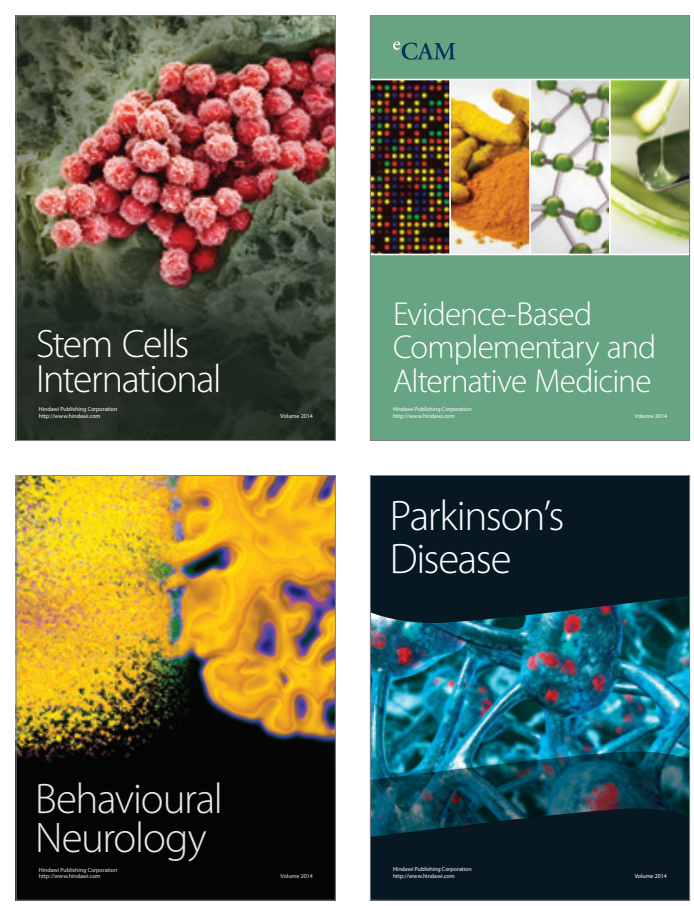

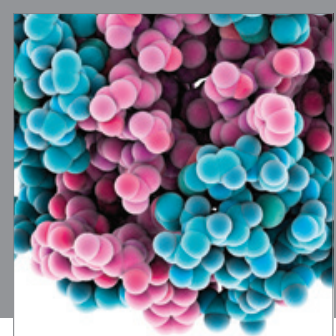

Journal of
Diabetes Research

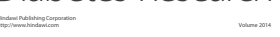

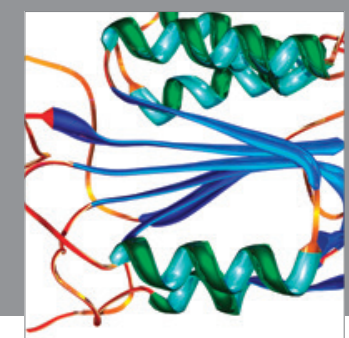

Disease Markers
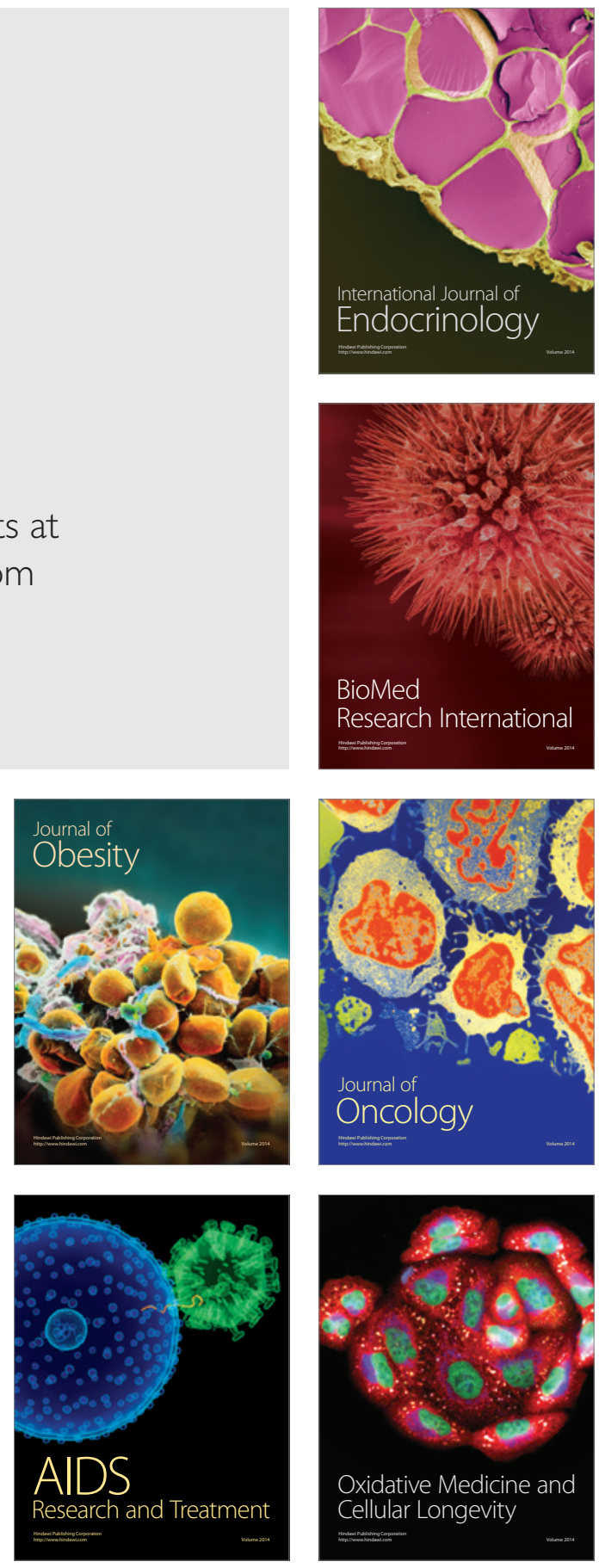